\title{
EL PISCO COMO CAPITAL CULTURAL DE MOQUEGUA
}

\author{
Wilfredo Kapsoli Escudero y Juan Rodríguez Pantigoso
}

\begin{abstract}
RESUMEN
La costa peruana por su divina situación geográfica y clima envidiable y seductor, permite que las uvas logren su grado máximo de dulzor y el pisco puede ser de calidad inigualable en toda latitud, por la cual se ha convertido en el producto bandera de nuestra patria por la que es exportado a las principales ciudades del mundo. Por eso, en el orbe entero, por ser fruto natural de las entrañas de la tierra madre, no hay un pisco como el nuestro ni habrá tampoco. Por eso es considerado como una exquisitez que arranca elogios de quienes lo degustan alegremente cuando de probar licores se trata. El pisco nació peruano, lo certifican todos nuestros viejos viñedos de ancestral historia que adornan la costa peruana y los miles de vitivinicultores que han trabajado los viñales.
\end{abstract}

Palabras Clave: Capital cultural, ruta del pisco, Moquegua.

\section{CULTURAL CAPITAL AS THE PISCO MOQUEGUA}

\section{ABSTRACT}

The Peruvian coast for its divine geographical location and an enviable and seductive climate allows the grapes to reach their maximum degree of sweetness and pisco may be and excellent quality in every latitude, for which it has become the flagship product of our country why it is exported to major cities worldwide. So in the whole world, being the natural fruit of the womb of mother earth, there is no pisco like ours nor there. Therefore it is considered in the universal orb as a delicacy that starts praise of those who tasted it happily when it comes liquor test. Peruvian pisco was born, I certify all our old vineyards of ancestral history adorning the Peruvian coast and the thousands of winemakers who have worked viñales.

KEYWORDS: Cultural Capital, route of pisco, Moquegua 


\section{La ruta del pisco}

I recorrer esta ruta encontraremos reservas vitivinícolas, en las que se ofrecen
los piscos codiciados. En torno a los amplios viñedos, se erigen añejas bodegas
coloniales, donde es posible apreciar el proceso de destilación y las enormes
barricas donde reposa el preciado licor. Hoy esta campiña, con sus aliados estratégicos, busca trabajar con nuevas tecnologías, las mismas que permitan ampliar los mercados. Éste es un ejemplo de cómo la minería puede sembrar agricultura de exportación.

Moquegua, ubicado al Sur del Perú, es un lugar privilegiado para quienes desean sustraerse de los caminos excesivamente transitados y descubrir, en cambio, los secretos de esta inesperada y sorprendente región. La calidad de sus tierras y sus inmejorables condiciones climáticas, que brindan un sol abrasador, han hecho posible que este terruño se convierta en un espacio propicio para el crecimiento de la uva, planta divina, cuyo fruto permite destilar los mejores piscos.

La historia es testigo que la existencia de la vid data de la llegada de los primeros españoles, que decidieron asentarse en el valle encantador. También los escritos de Garcilaso de la Vega aseguran que la cepa de esta planta llegó del Lejano Oriente como resultado de un error. Pero, lo cierto es que los primeros agricultores cimentados en la campiña, de comprobada fertilidad, se volcaron a preparar los campos y alcanzaron un notable desarrollo en el cultivo y producción de sus derivados.

Por la gran especialización alcanzada, incluso han sido reconocidos como los verdaderos y mejores productores. En su momento de mayor apogeo consiguieron cultivar hasta 1200 hectáreas de vid, con las que abastecían de materia prima a las bodegas existentes en la zona. Desde estas tierras, con el tiempo se extendió a todos los valles costeros de la patria (Lima e Ica) u otras localidades, donde había un clima aparente como para que prospere la vitivinicultura.

\section{Socios y aliados}

En la actualidad, los productores moqueguanos cultivan más de 200 hectáreas de vid y muchos de ellos han optado por modernizar el sistema de cultivo teniendo un uso eficiente del agua. La asociatividad entre la empresa minera de capitales ingleses, Anglo American Quellaveco, la Municipalidad Provincial de Mariscal Nieto y diversos productores, poco a poco van mostrando resultados alentadores, mejorando productos, desarrollando capacidades y abriendo nuevos mercados que generan progreso.

Actualmente la agroexportación es uno de los rubros de proyección ascendente en la economía peruana. Las alianzas estratégicas con empresas privadas han significado un factor determinante para posicionar nuestros productos en diversos mercados del mundo. Con ello, 


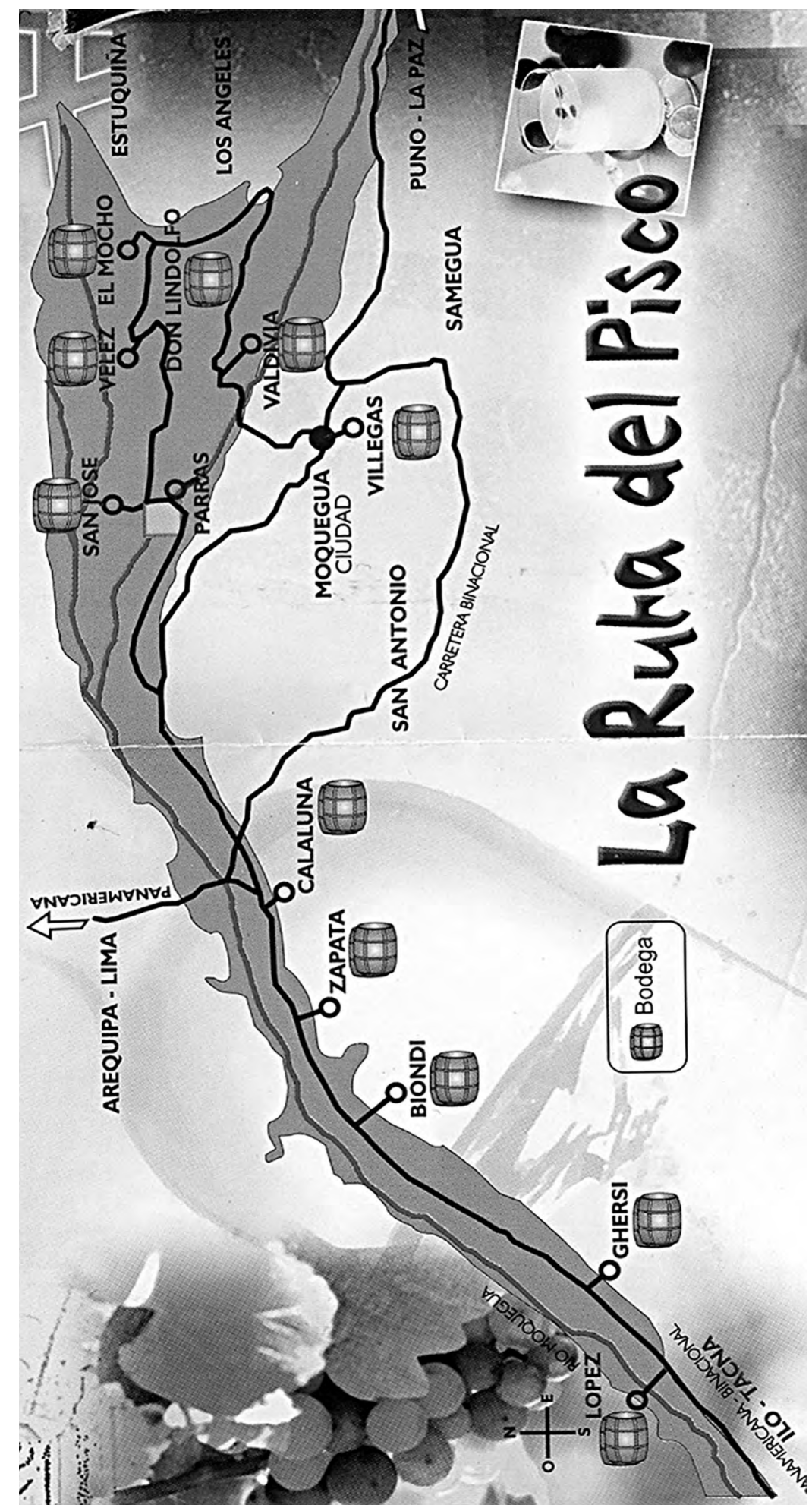


muchas regiones del país han dado un gran salto hacia la modernización, sacándole la vuelta a la pobreza. La sensación que Moquegua da es que algo grande está por llegar, su gente, sus productos y en especial su exquisito pisco, dejan ver cómo se preparan para un gran cambio. La región camina a pasos agigantados como se desprende de la siguiente encuesta:

\section{Las principales bodegas}

\begin{tabular}{|l|c|c|}
\hline Bodega & Frecuencia & $\%$ \\
\hline Biondi & 55 & 13 \\
\hline El Mocho & 50 & 12 \\
\hline Bodega Parras & 45 & 11 \\
\hline Bodega Reyes & 41 & 10 \\
\hline Vélez & 27 & 7 \\
\hline Don Lindolfo & 24 & 6 \\
\hline Valdivia & 23 & 6 \\
\hline Zapata & 21 & 5 \\
\hline Villegas & 18 & 4 \\
\hline Bodega Norvill & 17 & 4 \\
\hline Cornejo & 16 & 4 \\
\hline Jiménez & 14 & 3 \\
\hline El Campano & 11 & 3 \\
\hline Ghersi & 11 & 3 \\
\hline Paredes & 10 & 2 \\
\hline San José & 8 & 2 \\
\hline López & 6 & 1 \\
\hline Rayito de Sol & 4 & 1 \\
\hline Meléndez & 3 & 1 \\
\hline Otros & 11 & 3 \\
\hline
\end{tabular}

En el cuadro que antecede observamos que la bodega Biondi es la que más preferencia tiene entre los encuestados con un 13\%. Esto es comprensible toda vez que el pisco que produce esta fábrica está destinado al mercado internacional donde goza de mucha aceptación. El segundo lugar de preferencias ocupa la bodega El Mocho con 12\%, es decir, a solo un punto de la bodega Biondi. La razón de este porcentaje es que los piscos que produce este establecimiento es de plena aceptación de los propios lugareños, o sea, moqueguanos y foráneos residentes en dicha ciudad. El tercer lugar ocupa la bodega Parras con un 11\%, a sólo 1 punto de diferencia de la bodega El Mocho. Siguen en importancia las bodegas Reyes, Vélez, Don Lindolfo, Valdivia y otros.

\section{Las bodegas de pisco}

En el siguiente cuadro se muestra las bodegas del pisco más representativas y éstas son: Biondi en el primer lugar de preferencia con un 32\%, seguido de El Mocho con un $20 \%$ de 
representatividad y en un tercer lugar se ubican las bodegas Parras y Reyes con un $18 \%$. Otras bodegas como Norvill, Jiménez, Cornejo, Lindolfo, Villegas, entre otras, ocupan lugares inferiores a dichos porcentajes.

\begin{tabular}{|l|c|c|}
\hline Bodega & Frecuencia & $\%$ \\
\hline Biondi & 61 & 32 \\
\hline El Mocho & 38 & 20 \\
\hline Parras y Reyes & 33 & 18 \\
\hline Norvill & 10 & 5 \\
\hline Jiménez & 9 & 5 \\
\hline Cornejo & 6 & 3 \\
\hline Lindolfo & 6 & 3 \\
\hline Villegas & 5 & 3 \\
\hline Vélez & 3 & 2 \\
\hline El Campano & 2 & 1 \\
\hline Valdivia & 2 & 1 \\
\hline Bodega Omo & 2 & 1 \\
\hline Otros & 11 & 6 \\
\hline
\end{tabular}

\section{Las marcas más representativas}

\begin{tabular}{|l|c|c|}
\hline Bodega & Frecuencia & $\%$ \\
\hline Biondi & 50 & 38 \\
\hline Parras y Reyes & 19 & 15 \\
\hline Jiménez & 15 & 12 \\
\hline Mocho & 10 & 8 \\
\hline Norvill & 6 & 5 \\
\hline Mendoza & 4 & 3 \\
\hline Pisco Italia Vargas & 4 & 3 \\
\hline Villegas & 2 & 2 \\
\hline Paredes & 2 & 2 \\
\hline Vélez & 2 & 2 \\
\hline Pisco puro & 2 & 2 \\
\hline Mosto verde & 2 & 2 \\
\hline Zapata & 2 & 2 \\
\hline Otros & 10 & 8 \\
\hline
\end{tabular}

En este cuadro se muestra las marcas de pisco más representativas ubicando a Biondi nuevamente como primer lugar, con un 38\%. También podemos apreciar que la marca Parras y Reyes tiene un 15\% y ocupa un segundo lugar, como una marca de preferencia de los lugareños; seguidamente la marca Jiménez con un 12\% que indica su preferencia y reconocimiento a estas marcas entre los 3 primeros lugares. Seguidamente, las siguientes marcas ocupan lugares inferiores a estos porcentajes: El Mocho, Norvelli, Mendoza, Pisco Italia Vargas, entre otros. 


\section{Tipos de pisco de mayor preferencia}

\begin{tabular}{|l|c|c|}
\hline Variedad & Frecuencia & $\%$ \\
\hline Acholado & 27 & 20 \\
\hline Pisco Italia & 18 & 13 \\
\hline Pisco Puro & 17 & 13 \\
\hline Biondi & 10 & 7 \\
\hline Pisco Aromático & 10 & 7 \\
\hline Pisco Acholado & 9 & 7 \\
\hline Mosto verde & 6 & 4 \\
\hline Pisco Morro Verde & 4 & 3 \\
\hline Campano & 4 & 3 \\
\hline Semiseco & 4 & 3 \\
\hline Parras & 3 & 2 \\
\hline No Aromático & 3 & 2 \\
\hline Jiménez & 3 & 2 \\
\hline Mocho & 2 & 1 \\
\hline Alvilla & 2 & 1 \\
\hline Quebranta & 2 & 1 \\
\hline Pisco Parras y Reyes & 2 & 1 \\
\hline Otros & & \\
\hline
\end{tabular}

En el cuadro podemos apreciar que entre los piscos de mayor preferencia se encuentran: en primer lugar, el pisco Acholado con un $20 \%$ de aceptación en la población, seguido de Pisco Italia, con un buen porcentaje aunque un tanto distante del pisco Acholado, 18\%, y de igual aceptación, con un $17 \%$ el Pisco puro; lo que nos indica que el pisco Acholado es el preferido por los moqueguanos, seguido de los demás piscos que también tienen sus porcentajes de aceptación (Biondi, Pisco Aromático, Mosto verde, Morro verde, Campano, Semiseco, Parras, etc.).

\section{El pisco debe tomarse preferentemente}

\begin{tabular}{|l|c|c|}
\hline Formas de beber & Frecuencia & $\%$ \\
\hline Puro & 40 & 63 \\
\hline Gaseosa & 20 & 32 \\
\hline Otros & 3 & 5 \\
\hline
\end{tabular}

En el cuadro anterior observamos que la recomendación de los encuestados a la forma en que debe tomarse el pisco es que debe ser puro, con un 63\% de aceptación. Un $32 \%$ de encuestados respondió que el pisco debe tomarse con gaseosa y con otras bebidas en un $5 \%$. 


\section{Pisco sour debe constituir parte de las ceremonias sociales}

\begin{tabular}{|l|l|l|}
\hline Sí & 63 & $93 \%$ \\
\hline No & 5 & $7 \%$ \\
\hline
\end{tabular}

Aquí la mayoría de encuestados afirmó que el Pisco sour debe constituir parte de las ceremonias histórico-sociales con un $93 \%$, con un $7 \%$ que opina lo contrario.

\section{7. ¿Con qué tipo de pisco es mejor preparar el Pisco sour?}

\begin{tabular}{|l|c|c|}
\hline Marca & Frecuencia & $\%$ \\
\hline Acholado & 11 & 23 \\
\hline Pisco Puro & 8 & 17 \\
\hline Biondi & 7 & 15 \\
\hline Pisco Italia & 7 & 15 \\
\hline Italiano Aromático & 3 & 6 \\
\hline Pisco de Omate & 3 & 6 \\
\hline Otros & 8 & 17 \\
\hline
\end{tabular}

En el cuadro observamos que un $23 \%$ de encuestados opina que el mejor pisco para preparar el Pisco sour es el Acholado. Muy cerca se ubica el Pisco puro con un $17 \%$ y un $15 \%$ opta por el Pisco Biondi y Pisco Italia. Seguido de otros piscos que cuentan con una menor preferencia (Pisco Italiano aromático, Pisco de Omate, otros).

\section{8. ¿Cuáles son los piscos de exportación?}

\begin{tabular}{|l|c|c|}
\hline Marca & Frecuencia & $\%$ \\
\hline Biondi & 39 & 40 \\
\hline Pisco Acholado & 8 & 8 \\
\hline Jiménez & 8 & 8 \\
\hline Mocho & 7 & 7 \\
\hline Pisco Italia & 6 & 6 \\
\hline Parras Verde & 5 & 5 \\
\hline Pisco Aromático & 4 & 4 \\
\hline Norvill & 3 & 3 \\
\hline Pisco puro Italia & 3 & 3 \\
\hline Italiano no aromático & 2 & 2 \\
\hline Pisco Biondi & 2 & 2 \\
\hline Parras y Reyes & 2 & 2 \\
\hline Otros & 8 & 8 \\
\hline
\end{tabular}


En el cuadro que antecede observamos que la mayoría de los encuestados, $40 \%$, opina que el pisco de exportación moqueguano es el pisco Biondi. Con un segundo lugar están los piscos Acholado y el pisco Jiménez con un 8\%, y en tercer lugar el pisco El Mocho, con un 7\%, muy cerca del segundo lugar. Luego mencionan a otros piscos de exportación, entre los que tenemos a: Pisco Italia, Parras Verde, Pisco Aromático, Norvill, Pisco puro Italia, entre otros.

\section{9. ¿Conoce alguna poesía, canción o tradición dedicada al pisco?}

\begin{tabular}{|l|l|l|}
\hline No & 55 & $82 \%$ \\
\hline Sí & 12 & $18 \%$ \\
\hline
\end{tabular}

En el cuadro observamos que el $82 \%$ de los encuestados desconoce alguna poesía, canción o tradición dedicada al pisco. Una minoría de 18\% de encuestados indicó conocer algunas letras que manifestaron recordar. Tales como:

Pisco, licor bendito, qué haces afuera que no vienes adentro

¿Quéyo soy un bebedor? Eso nunca ni lo sueñes, ni acusarme, te empeño, mas yo pruebo

lo mejor, y no bebo por amor y si mi suerte es bien negra

* Moquegua querida tierra de los piscos y vinos, invitamos que brindan y conozcan lo que tenemos es puro

* El pisco de nuestro suelo es por darnos luz de vida emblemática, bebida que se toma hasta en el cielo. Por eso le tienen celo al ser trago soberano

10. ¿El pisco preferible consumirlo como?

\begin{tabular}{|l|c|c|}
\hline Modalidad & Frecuencia & $\%$ \\
\hline Aperitivo & 55 & $82.0 \%$ \\
\hline Después de las comidas & 11 & $16.4 \%$ \\
\hline Con las comidas & 1 & $1.4 \%$ \\
\hline
\end{tabular}

En el cuadro se observa que un $82.09 \%$ de encuestados indica que el pisco es preferible consumirlo como aperitivo, seguido de un $16.42 \%$ que opina que es mejor consumirlo después de las comidas, y el 1.49\% opina que es más recomendable con las comidas. 


\section{1. ¿Cómo reconocer un buen pisco?}

Como todo licor, un pisco de calidad, se reconoce por su sabor y aroma; sin embargo, existe una prueba que no falla y que está al alcance de todos, por la cual se puede determinar qué de bueno tiene el pisco que se quiere probar. Esta extraña figura que se forma dentro de la botella se le ha bautizado como "Cordón y Rosa". Es muy sencilla de realizar tanto para conocedores como consumidores habituales que puede llegar a conocer si es verdad tanta belleza.

Primero hay que batir con energía la botella de un pisco del bueno, en círculos, y debe quedar una viscosidad como aceite girando al centro del recipiente en forma de un torbellino. A la cola de burbujas distribuidas a lo largo se le denomina "Cordón"y a las burbujas que flotan sobre el ojo del torbellino se les llama "Rosa". Si es así, puede decir ¡Salud! con toda confianza y deleitarse con tan divino licor y rendir culto a la existencia humana. Por ser lágrimas candentes de las parras cuajadas en comunión celestial entre tierra, agua y sol, en esta patria de ensueño y tradición, es tan peruano como este hermoso y generoso suelo que nos cobija y que es testigo de nuestra grandeza y proyección al futuro a pasos agigantados. ¡Eso es el pisco señores!

La costa peruana por su divina situación geográfica y un clima envidiable y seductor, permite que las uvas logren su grado máximo de dulzor y el pisco pueda ser de calidad iniguadable en toda latitud, por la cual se ha convertido en el producto bandera de nuestra patria por la que es exportado a las principales ciudades del mundo. Por eso, en el orbe entero, por ser fruto natural de las entrañas de la tierra madre, no hay un pisco como el nuestro ni habrá tampoco. Por eso es considerado en el orbe universal como una exquisitez que arranca elogios de quienes lo degustan alegremente cuando de probar licores se trata. El pisco nació peruano, lo certifican todos nuestros viejos viñedos de ancestral historia que adorna la costa peruana y los miles de vitivinicultores que han trabajado los viñales.

\section{Referencias}

PINTO, Ismael. Antología de Moquegua, Lima, 1975.

WISE, Karen. Moquegua. Ed. Museo Contisuyo, 1998. 


\title{
ANEXO 1
}

\section{Las bodegas de la época colonial de Moquegua}

\author{
Por: Prudence M. Rice
}

El asentamiento colonial español del valle del Osmore se estableció a mediados del siglo XVI y, por tradición, el 25 de noviembre de 1541, día de la consagración de la Iglesia de Santa Catalina, se conoce como la fecha de la fundación de Moquegua. Los archivos de esta ciudad no cuentan con documentos procedentes de las primeras décadas de ocupación, tampoco se conoce los nombres de los fundadores de la ciudad. El primer historiador de Moquegua, Juan Antonio Montenegro y Ubalde (1782-1854), señala que trece individuos fundaron la ciudad aunque sólo se conoce el nombre de uno de ellos: Pedro Cancino de España.

El valle de Osmore fue el límite entre las dos audiencias, o distritos administrativos, que gobernaban el sur del Perú. Dos comunidades que se establecieron cerca de la confluencia de los tres tributarios del Osmore, los ríos Tumilaca, Huaracane y Torata, constituyeron los principales asentamientos de estos distritos. En la margen derecha u occidental del río, perteneciente a la Audiencia de Lima, se encontraba la ciudad de San Sebastián de Escapagua, ubicada aproximadamente donde en la actualidad se encuentra el aeropuerto de Moquegua, en Alto de la Villa. La margen izquierda u oriental pertenecía a la Audiencia de Charcas, conjuntamente con el pueblo que más adelante se conoció con el nombre de Santa Catalina de Guadalcázar, Valle de Moquegua o simplemente Moquegua.

El asentamiento español se extendió a lo largo del valle de Osmore. Existen pruebas de la ocupación de los españoles en las partes más altas del valle, ya que se han encontrado objetos de procedencia europea tales como loza, cuentas y monedas en Torata y Camata. Se construyó un templo en Torata antes que el sitio fuera abandonado y se trasladara a las partes más bajas del valle alto en los alrededores del río Torata. También se encontró restos arqueológicos del asentamiento de la época colonial cerca de llo. Sin embargo, Moquegua fue el principal centro económico y demográfico del Osmore en la época Colonial temprana. La población de Moquegua en 1628 fue de 14440 habitantes, mientras que el censo de 1792 mostró 8323 habitantes en la ciudad y 28801 en la provincia.

Cuando los españoles llegaron a Moquegua, al igual que en otras áreas del Nuevo Mundo, trajeron con ellos nuevas ideologías (como el catolicismo), nuevas tecnologías, y nuevas comodidades desde sus hogares. Los cultivos importados incluyeron un grupo de plantas y animales característicos de la Península Ibérica, tales como uvas, olivos, trigo, vacas, cabras, ovejas, cerdos y caballos, azúcar, alfalfa y mulas, entre otros. Muchas de las plantas y animales traídos desde España se establecieron rápidamente en Moquegua, creciendo en los diversos climas y elevaciones encontradas a lo largo de la costa y sierra. Se sabe, por ejemplo, que en la época Colonial temprana los olivos crecieron en las regiones costeras alrededor de llo, las uvas predominaron en el valle medio y el trigo se cultivó 
en los valles altos, en las inmediaciones de Torata. Antonio Vázquez de Espinosa estuvo en Moquegua en 1618 o 1619 y la describe como una rica zona agrícola productora de uva, membrillo, melocotón, higo, melón, caña de azúcar, trigo, maíz, garbanzo, habas y ají, entre otros.

La economía del valle medio de las cercanías de Moquegua, dependía mayormente del cultivo de la uva y de la producción de vino y pisco. Los primeros relatos sobre el cultivo de la vid en este valle datan de 1587, aunque es probable que el cultivo de este fruto se haya iniciado con anterioridad. Los documentos de archivo registran el establecimiento de viñedos y envíos de vino y pisco a comunidades de la sierra a partir de fines del siglo XVI. Entre los viñedos que se mencionan en estos documentos tenemos Cupina, Omo, Corpanto, Yaravico, Locumbilla y Estopacaje. Aún hay lugares que llevan estos nombres en el valle de Moquegua.

Los historiadores han reconocido la importancia económica de la viticultura en Moquegua durante la época Colonial, pero los datos adicionales que proporcionan las investigaciones arqueológicas dirigen su atención hacia la verdadera magnitud de la industria vitivinícola. Por ejemplo, las prospecciones y excavaciones arqueológicas llevadas a cabo por el Proyecto Bodegas de Moquegua (dentro del Programa Contisuyo), entre los años 1985 y 1989, revelaron la existencia de 130 bodegas a lo largo de $28 \mathrm{~km}$ del valle medio. Estas bodegas se sitúan principalmente en las zonas escarpadas, sobre las llanuras irrigadas, demarcando los límites del valle hacia ambos lados. Muchos de estos sitios han sido dañados o destruidos por construcciones modernas o por la actividad urbana y, en la actualidad, se conoce algunas de estas bodegas sólo a través de los restos de objetos desperdigados por la superficie del terreno o mediante la tradición oral. Sin embargo, otras han sobrevivido siglos y están en condiciones bastante aceptables, siendo éstas a las que el Proyecto Bodegas prestó mayor atención. Algunas bodegas como, por ejemplo, Chincha y Sacatita en el valle bajo, Locumbilla en el valle medio y Yahuay y Huaracane en el valle norte, aún tiene sus paredes originales y muchos otros detalles permanecen intactos. Arquitectónicamente, las bodegas pueden ser descritas como complejos de una sola pieza, con techos de caña y paredes de adobe conformando cuartos contiguos y patios abiertos. Para fines prácticos están divididas en dos áreas: un área "industrial" donde se llevan a cabo las actividades relacionadas con la elaboración de vino; y otra área reservada como vivienda para los propietarios (o con mayor frecuencia los administradores) y los trabajadores. Por lo menos tres bodegas cuentan con capillas cerca de las habitaciones. Los patios habrían servido para guardar equipo, como zona reservada para los animales y como áreas de estacionamiento para la carga y descarga de los contenedores de vino. Las excavaciones efectuadas en y alrededor de las áreas residenciales de las bodegas, proporcionan una visión de la vida colonial entre los siglos XVI y XVIII. Durante las excavaciones recuperamos una variedad de restos de plantas y animales, dentro de las que se incluye tanto aportes europeos (semillas de uvas, aceitunas y melocotones y huesos de ganado vacuno) como plantas y animales indígenas (maíz, calabaza, frijol y huesos de 
llama y alpaca). Entre los objetos encontrados podemos citar una variedad de ceramios usados en la preparación y almacenaje de alimentos, así como vidrios y utensilios de madera; también madera y metal como materiales de construcción para mampostería; prendas de vestir y ornamentos (botones, fragmentos de telas), implementos para costura, juguetes, y restos de armas y equipo para caballos. Resulta interesante saber que pocos de estos objetos fueron traídos desde España o Europa, por el contrario, parecen ser de manufactura andina. La escasez de productos europeos sugiere que Moquegua fue una región alejada y aislada durante la mayor parte de la época de la Colonia.

En los sectores industriales de las bodegas existen diversas estructuras y rasgos característicos relacionados con la elaboración del vino. Estos se encuentran dispuestos en las laderas de los viñedos para aprovechar la gravedad durante las fases de elaboración del vino. Por ejemplo, los lagares de piedra alineados, o tinas para apisonar la uva estaban ubicados generalmente sobre y en la parte trasera del complejo. Se traía la uva desde el campo, se depositaba en las tinas y luego era pisada para extraer su jugo. Este jugo pasaba a través de drenajes ubicados en la base de la parte más baja de los lagares y luego era canalizado hacia contenedores para su fermentación y almacenamiento. Estas operaciones se llevaban a cabo en enormes vasijas de barro no vidriado Ilamadas tinajas. Estas tinajas eran alineadas y parcialmente enterradas en el piso de cuartos largos, angostos y oscuros. Para la elaboración de productos destilados, tales como aguardiente y pisco, se sacaba el vino de las tinajas (supuestamente con la ayuda de una bomba) y se colocaba en otro lagar tipo tanque cerca de la destilería o falca. Las falcas estaban generalmente ubicadas en la parte frontal o en el nivel más bajo de la bodega, y por el peligro de incendio del alambique, siempre eran situadas a una cierta distancia de las estructuras principales.

Además de estas estructuras e instalaciones, algunas bodegas tenían hornos como parte de sus sectores industriales. Se encontró 26 hornos que tenían relación con las bodegas de Moquegua, todos ellos de diversos tamaños y formas. Estos hornos eran usados en la cocción de tinajas y pequeñas jarras que eran utilizadas para el transporte de vino, llamadas botijas, y probablemente también se hayan usado para la calcinación de cal.

Se calcula que si las 130 bodegas operaban al mismo tiempo, más de tres millones de galones de vino y pisco podrían haber sido almacenados en las tinajas de Moquegua. Esta cantidad de vino no era consumida en su totalidad por los habitantes de Moquegua; sino que la enviaban a las comunidades de la sierra, como del lago Titicaca y a la minas de plata de Potosí en el Alto Perú (hoy Bolivia). Se dice que Moquegua abasteció entre $60 \%$ y $90 \%$ del pisco y vino que se consumía en los distritos mineros y que la economía de Moquegua sufrió las mismas alzas y altibajos de las minas. Además, algunos historiadores han notado que debido a que en las tierras de Moquegua se cultivaba prácticamente sólo uvas, esta zona carecía de alimento para sus propios habitantes. Muchas veces los pobladores de Moquegua dependían de zonas surtidoras como el valle de Torata, que los abastecía de trigo, fruta y vegetales. 
Aproximadamente 300 años después de su inicio en el valle de Moquegua, la industria del vino sufrió un colapso, hacia fines de 1800. Las causas fueron numerosas y dentro de ellas se puede citar los terremotos, la guerra y la filoxera. Por lo menos una docena de fuertes terremotos han sido registrados en la historia de Moquegua, el primero de ellos relacionado con la erupción del volcán Huaynaputina en febrero de 1600. Asimismo, el terremoto de agosto de 1868 ocasionó una gran destrucción y crisis económica en el sur del Perú, y en Moquegua las construcciones se vinieron abajo y las tinajas se rompieron perdiendo su contenido. Aproximadamente una década después se inicio la Guerra del Pacífico que enfrentó a Perú y Chile. Moquegua fue invadida cuatro veces entre 1879 y 1883 y los chilenos fueron responsables de desorganizar el comercio del vino con la sierra, así como de destruir las propiedades. Finalmente, durante el mismo periodo, los viñedos de Moquegua sufrieron un ataque de filoxera, un áfido que ataca las raíces de la parra de uva ocasionando su muerte. El resultado de todos estos desastres fue la ruina de la industria del vino a principios del presente siglo.

En la actualidad, las tierras que se usaron para el cultivo de la vid, se emplean para pastar ganado o para el cultivo de alfalfa, hortalizas, maíz, papas, entre otros. De acuerdo con un estudio realizado a mediados de la década de 1970, menos del $2 \%$ de la tierra del valle de Moquegua se dedicaba al cultivo de la vid y sólo cuatro de las bodegas se encuentran operando en la actualidad, las que se dedican a la producción de una variedad de vinos y productos destilados, entre los cuales el más importante es el pisco. 


\section{ANEXO 2}

Otros comentarios en relación con el pisco moqueguano:

- $\quad$ El pisco moqueguano es agradable.

- Es uno de los piscos aromáticos por excelencia.

- Para algunos es el mejor del Perú.

- El pisco moqueguano debe ser más difundido, y que se dé a conocer en el Perú.

- Deberá difundir más las marcas, hacer marketing.

- La calidad de nuestro pisco es único y que todas las bodegas deben juntarse para ser exportado representando Moquegua.

- Moquegua es la tierra de los piscos y vinos incomparables por su calor, clima; necesitamos unirnos para exportar hacia los mercados europeos y asiáticos.

- Probarlo con moderación que es delicioso.

- Tenemos una gran materia y variedad para ser exportada y dará así la generación de pequeñas y medianas empresas.

- El pisco en el valle de Moquegua se elaboró inicialmente con una uva misión y/o negra criolla que debe ser fruto de la reproducción sexual de la uva negra.

- Generalmente el pisco no es tan consumido en Moquegua, quien consume pisco es alguien que conoce.

- Este tema debe ser tocado de manera amplia y detallada en todos los medios de comunicación masivos de la localidad y de fuera de la localidad. 